\title{
RELAÇÃO ENTRE A VARIABILIDADE ESPACIAL DAS FRAÇÕES GRANULOMÉTRICAS DO SOLO E A PRODUTIVIDADE DO FEIJOEIRO SOB PLANTIO DIRETO(1)
}

\author{
Aline Emy Kitamura ${ }^{(2)}$, Morel de Passos e Carvalho ${ }^{(3)}$ \\ \& Cesar Gustavo da Rocha Lima ${ }^{(2)}$
}

\begin{abstract}
RESUMO
A distribuição granulométrica do solo exerce grande influência na produtividade vegetal. No ano agrícola de 2002/2003, foram analisados atributos da planta: produtividade de grãos (PG) e da palha (PP) do feijoeiro; e do solo: teores de argila, silte e areia de um Latossolo Vermelho distroférrico sob plantio direto, do Campus Experimental da Faculdade de Engenharia de Ilha Solteira/UNESP, em Selvíria (MS). O objetivo foi analisar a variabilidade dos atributos pesquisados, de forma a caracterizar suas dependências espaciais e as correlações, linear e espacial, entre eles. Foi instalada uma malha experimental para coleta dos dados, com 135 pontos amostrais, distribuídos numa área total de $8.000 \mathrm{~m}^{2}(160 \times 50 \mathrm{~m})$. A variabilidade dos dados foi baixa para o teor de argila, média para os teores de silte e areia e alta para PP e PG. A maioria dos atributos apresentou moderada dependência espacial, com alcance entre 19,8 m (silte) e 103,1 m (areia) e de 29,8 m para a PG. Foi observada evidente correlação espacial entre todos os que apresentaram, dois a dois, os maiores coeficientes de correlação; entretanto, entre aqueles que apresentaram os menores, os dados sugeriram, em alguns casos, também haver concordante correlação espacial.
\end{abstract}

Termos de indexação: geoestatística, dependência espacial, atributos físicos do solo, textura do solo.

\footnotetext{
(1) Trabalho de mestrado desenvolvido no Programa de Pós-Graduação em Sistemas de Produção da Faculdade de Engenharia de Ilha Solteira - UNESP/Campus de Ilha Solteira. Recebido para publicação em agosto de 2004 e aprovado em novembro de 2006. (2) Mestrando da Faculdade de Engenharia, Universidade Estadual Paulista - UNESP. Campus de Ilha Solteira. Caixa Postal 31, CEP 15385-000 Ilha Solteira (SP). E-mails: alineemy@bio.feis.unesp.br; etaugustus@aluno.feis.unesp.br

(3) Professor Adjunto, Departamento de Fitossanidade, Engenharia Rural e Solos. Faculdade de Engenharia, UNESP. E-mails: morel@agr.feis.unesp.br; mpcarva53@yahoo.com.br
} 


\title{
SUMMARY: RELATIONSHIP BETWEEN THE SPATIAL VARIABILITY OF PARTICLE-SIZE FRACTIONS OF SOIL AND COMMON BEAN YIELD UNDER NO-TILLAGE
}

\begin{abstract}
The soil particle-size distribution has great influence on the plant yield. In the 2002/ 2003 growing season, plant and soil attributes of an experimental station of the Faculdade de Engenharia/UNESP (Ilha Solteira/SP - Brazil) were analyzed: common bean grain yield $(G P)$, straw yield (SP), clay content (CL), silt (SI) and sand (SA) of a Red Latosol (Oxisol) under no-tillage. The objective was to analyze the variability of the evaluated attributes to characterize spatial dependences and to study linear and spatial correlations between them. An experimental grid for data collection was installed with 135 points in an area of $8.000 \mathrm{~m}^{2}$. The data variability was low for $C L$, intermediate for SI and SA, and high for SP and GP. The majority of the attributes presented moderate spatial dependence, ranging from $19.8 \mathrm{~m}$ (SI), to $103.1 \mathrm{~m}$ (SA), and $29.8 \mathrm{~m}$ for the GP. An evident spatial correlation was observed between all those variables that presented, two by two, the highest correlation coefficients. However, among the lowest ones, the data suggested, in some cases, a similar spatial correlation.
\end{abstract}

Index-terms: geostatistics, spatial dependence, soil physical attributes, soil texture.

\section{INTRODUÇÃO}

A preocupação com o meio ambiente vem crescendo cada vez mais, e com ela a necessidade de estudos detalhados, visando à implantação do seu planejamento conservacionista, com o objetivo de manejar de forma adequada seus recursos naturais, agredindo-o o mínimo possível. O conhecimento dos atributos físicos dos solos, envolvendo principalmente aqueles relacionados com sua distribuição granulométrica e que influenciam diretamente o fluxo superficial e o movimento de água no solo, é fundamental para o planejamento ambiental. É essencial também o estudo de sua fertilidade, uma vez que a baixa disponibilidade de nutrientes é um fator limitante ao rendimento adequado das culturas. Por sua vez, o maior rendimento agrícola proporciona aumento da massa vegetal, que futuramente será convertida em resteva vegetal (palha), constituindo-se num dos fatores controladores da erosão (Guerra, 1998).

A textura do solo tem grande influência no manejo da irrigação, pois está relacionada com a capacidade de retenção de umidade, a permeabilidade e o seu preparo. Embora a capacidade de retenção de água e nutrientes esteja relacionada com outras características, como estrutura, conteúdo de matéria orgânica, tipo de argila e de cátions, possui boa relação com os teores de argila, aumentando, em geral, proporcionalmente a estes. De modo geral, os solos com elevados teores de argila possuem faixa mais ampla de umidade, enquanto a dos arenosos é bem mais estreita.

De acordo com Vieira et al. (1988), outras características, como infiltração, permeabilidade às raízes e aeração, também são bastante influenciadas pela textura.
Com a preocupação de verificar em que situação pode ser utilizada a cokrigagem, ou seja, qual o nível de correlação necessário para que as estimativas sejam consistentes, Landim (1998) selecionou dados de penetração padronizada (SPT), obtidos no sítio urbano de Bauru (SP), a partir de 92 sondagens a percussão. Constatou-se que, à medida que os coeficientes de correlação espacial entre as variáveis diminuíram, os erros associados à estimação da variável que se quer estudar aumentaram. Esse resultado apontou para um cuidado especial que se deve ter com o nível de correlação entre as variáveis enfocadas, antes de se iniciar uma aplicação da cokrigagem.

A variabilidade espacial dos atributos do solo influencia de forma decisiva o manejo a ser adotado nas áreas cultivadas. O conhecimento da variabilidade das propriedades do solo é um importante passo para que seja efetuado o seu manejo adequado. Além da variabilidade natural do solo, as práticas agrícolas de manejo são fontes adicionais que a implementam. $\mathrm{O}$ manejo do solo pode afetar seus atributos químicos, físicos e biológicos, sendo muito importante, sobretudo, nas camadas superficiais (Bhatti et al., 1991). O conhecimento dessas variáveis é importante para seu levantamento e respectiva classificação, avaliação de sua fertilidade, desenvolvimento de esquemas de amostragem mais adequados, planejamento experimental, bem como para o seu manejo e recuperação (Souza, 1992).

A variabilidade espacial da granulometria tem sido estudada em várias classes de solos, tendo sido geralmente constatada a presença de dependência espacial, cujos alcances têm variado entre 15 e 10.500 m (Gonçalves, 1997; Souza et al., 1997; Vieira, 1997; Salviano et al., 1998; Zimback \& Cataneo, 1998; Gonçalves et al., 2000, 2001; Gonçalves \& Folegatti, 
2002; Araújo, 2002; Barbieri et al., 2002; Rabah, 2002; Roque et al., 2002). Portanto, os objetivos do presente trabalho foram: analisar a variabilidade e caracterizar a dependência espacial dos atributos granulométricos do solo e da produtividade de grãos do feijoeiro; e estudar as correlações, linear e espacial, entre tais atributos.

\section{MATERIAL E MÉTODOS}

O trabalho foi desenvolvido na Fazenda de Ensino e Pesquisa da Faculdade de Engenharia de Ilha Solteira - FEIS/UNESP, no município de Selvíria (MS), na latitude $22^{\circ} 23^{\prime} \mathrm{S}$ e na longitude $51^{\circ} 27^{\prime} \mathrm{W}$. A precipitação média anual é de $1.300 \mathrm{~mm}$ e a temperatura média, de $23,7^{\circ} \mathrm{C}$. O tipo climático é $\mathrm{A}_{\mathrm{w}}$, segundo a classificação de Köppen, caracterizado como tropical úmido com estação chuvosa no verão e seca no inverno (Demattê, 1980). O solo, reclassificado segundo o Sistema Brasileiro de Classificação de Solos (Embrapa, 1999), é um Latossolo Vermelho distroférrico típico muito argiloso, A moderado, hipodistrófico, álico, caulinítico, férrico, epicompactado, muito profundo, moderadamente ácido. No quadro 1 é apresentada a sua análise físico-química.
A área experimental, instalada sob pivô central, foi cultivada nos últimos seis anos para produção de grãos, normalmente sob o sistema de preparo convencional do solo ou sob semeadura direta, com as sucessões milho/feijão e milho/arroz. Este estudo foi conduzido no solo cultivado no sistema plantio direto. Realizou-se a dessecação do milho, que antecedeu a cultura do feijoeiro, com a aplicação de $1,8 \mathrm{~kg} \mathrm{ha}^{-1}$ (p.a.) do herbicida glyfosate entre 15 e 18/4/2002. Em 6/5/2002, foi semeada a cultivar de feijoeiro IAC Carioca Aeté, no espaçamento de 0,50 m entre linhas e com 14 sementes por metro de semeadura, conforme as recomendações agronômicas da cultura (Fahl et al., 1998). Na adubação de base foram utilizados $200 \mathrm{~kg} \mathrm{ha}^{-1}$ da fórmula 08-28-16. Na de cobertura, realizada 20 dias após a emergência das plântulas (30/4/2002), foram aplicados $200 \mathrm{~kg} \mathrm{ha}^{-1}$ da fórmula 20-00-20.

A grande malha experimental ficou constituída de cinco transeções paralelas ao eixo x, com 15 pontos de amostragem cada uma. Nesse caso, foi estabelecido um total de 75 pontos amostrais, com espaçamento entre pontos de $10 \times 10 \mathrm{~m}$, numa área total de $8.000 \mathrm{~m}^{2}(160 \times 50 \mathrm{~m})$. Foram, ainda, alocadas por sorteios, dentro da grande malha, mais quatro pequenas malhas de pontos amostrais, visando

Quadro 1. Análise físico-química do Latossolo Vermelho distroférrico de Selvíria (MS) que abrigou a malha experimental de campo

\begin{tabular}{|c|c|c|c|c|c|c|c|c|c|c|c|c|}
\hline \multicolumn{2}{|c|}{ Horizonte } & \multicolumn{5}{|c|}{ Composição granulométrica } & \multirow{2}{*}{\multicolumn{3}{|c|}{$\begin{array}{c}\text { Relação } \\
\text { Silte/argila }\end{array}$}} & \multirow{2}{*}{\multicolumn{3}{|c|}{ Densidade do solo }} \\
\hline \multirow[t]{2}{*}{ Símbolo } & \multirow{2}{*}{$\begin{array}{c}\text { Profundidade } \\
\mathrm{m}\end{array}$} & \multicolumn{2}{|l|}{ Areia } & \multicolumn{2}{|l|}{ Silte } & Argila & & & & & & \\
\hline & & 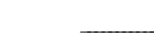 & 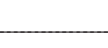 & $-\mathrm{g} \mathrm{kg}^{-1}$ & $\ldots$ & - & & & & & $\mathrm{kg} \mathrm{dm}$ & \\
\hline $\mathrm{Ap}$ & $0-0,15$ & 230 & & 120 & & 650 & & 0,1 & & & 1,30 & \\
\hline $\mathrm{A}_{3}$ & $0,15-0,30$ & 210 & & 120 & & 670 & & 0,1 & & & 1,20 & \\
\hline $\mathrm{B}_{21}$ & $0,30-1,00$ & 160 & & 130 & & 710 & & 0,1 & & & 1,20 & \\
\hline \multirow[t]{4}{*}{$\mathrm{B}_{22}$} & $1,00-1,75+$ & 160 & & 120 & & 720 & & 0,1 & & & 1,10 & \\
\hline & & \multicolumn{11}{|c|}{ Complexo sortivo } \\
\hline & & $\mathbf{P}$ & $\mathbf{C a}^{2+}$ & $\mathbf{M g}^{2+}$ & $\mathbf{K}^{+}$ & SB & $\mathrm{Al}^{3+}$ & $\mathbf{H}^{+}$ & CTC & $\mathrm{V}$ & m & MO \\
\hline & & $\mathrm{mg} \mathrm{dm}^{-3}$ & 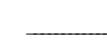 & $\underline{2}$ & $-\mathrm{r}$ & $\mathrm{lmol}_{\mathrm{c}} \mathrm{dm}^{-3}$ & 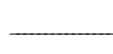 & 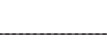 & - & $\underline{-}$ & - & $\mathrm{g} \mathrm{dm}^{-1}$ \\
\hline Ap & $0-0,15$ & 1,0 & 17,0 & 15,4 & 0,8 & 33,2 & 1,0 & 40,0 & 74,2 & 45 & 3 & 16,6 \\
\hline $\mathrm{A}_{3}$ & $0,15-0,30$ & 0,0 & 1,5 & 1,6 & 0,5 & 3,6 & 8,0 & 33,0 & 44,6 & 8 & 69 & 13,6 \\
\hline $\mathrm{B}_{21}$ & $0,30-1,00$ & 1,0 & 0,5 & 0,4 & 0,4 & 1,3 & 5,0 & 32,0 & 38,3 & 3 & 79 & 10,2 \\
\hline \multirow[t]{3}{*}{$\mathrm{B}_{22}$} & $1,00-1,75+$ & 0,0 & 1,0 & 0,8 & 0,5 & 2,3 & 2,0 & 28,0 & 32,3 & 7 & 47 & 4,1 \\
\hline & & \multicolumn{4}{|c|}{ pH } & \multicolumn{5}{|c|}{ Ataque sulfúrico } & & \\
\hline & & $\mathrm{H}_{2} \mathrm{O}$ & $\mathrm{KCl}$ & \multicolumn{2}{|c|}{$\Delta \mathbf{p H}$} & $\mathrm{SiO}_{2}$ & $\mathrm{Al}_{2} \mathrm{O}_{3}$ & \multicolumn{2}{|c|}{$\mathrm{Fe}_{2} \mathrm{O}_{3}$} & $\mathrm{TiO}_{2}$ & $\mathbf{K i}$ & $\mathbf{K r}$ \\
\hline & & & & & & \multicolumn{5}{|c|}{ dag $\mathrm{kg}^{-1}$} & & \\
\hline Ap & $0-0,15$ & 5,1 & 4,8 & $-0,3$ & & 20,60 & 15,17 & 28 & & 4,06 & 2,31 & 1,05 \\
\hline $\mathrm{A}_{3}$ & $0,15-0,30$ & 4,9 & 4,3 & $-0,6$ & & 21,35 & 16,98 & 26 & & 4,03 & 2,13 & 1,07 \\
\hline $\mathrm{B}_{21}$ & $0,30-1,00$ & 4,9 & 4,2 & $-0,7$ & & 23,58 & 32,28 & 28 & & 3,61 & 1,14 & 0,75 \\
\hline $\mathrm{B}_{22}$ & $1,00-1,75+$ & 5,3 & 4,6 & $-0,7$ & & 22,59 & 16,84 & 27 & & 3,67 & 2,28 & 1,10 \\
\hline
\end{tabular}


detalhar o estudo da dependência espacial dos dados para condições menores do que $10 \mathrm{~m}$. Cada pequena malha ficou posicionada entre quatro pontos amostrais da grande malha, constituindo-se de um ponto no seu centro e mais 14 pontos, com espaçamento entre eles de $2,5 \times 2,5 \mathrm{~m}$. Como cada uma delas adicionou mais 15 pontos amostrais, o número total destes foi de 135 . Dessa forma, para cada local de coleta dos dados de campo, nos respectivos locais de cada um dos 135 pontos amostrais da malha regular de coleta de dados, foram tomadas duas amostras simples do solo, referentes às camadas de zero a $0,10 \mathrm{~m} \mathrm{e} 0,10$ a $0,20 \mathrm{~m}$, destinadas à determinação da granulometria. Esse procedimento foi efetuado com o trado de caneca, com diâmetro interno de $0,08 \mathrm{~m}$ e altura de $0,20 \mathrm{~m}$. Foram efetuadas 270 análises granulométricas.

Os atributos pesquisados do solo foram argila (ARG), silte (SIL) e areia (ARE), identificados, da seguinte forma: (a) camada de zero a 0,10 m: ARG1, SIL1 e ARE1; e (b) camada de 0,10 a 0,20 m: ARG2, SIL2 e ARE2. O método utilizado foi o da pipeta, conforme recomendado por Embrapa (1997) e Gee \& Bauder (1986), sendo o agente químico dispersante o $\mathrm{NaOH} 1 \mathrm{~mol} \mathrm{~L}^{-1}$. Os atributos da planta foram as produtividade de grãos (PG) e a biomassa seca da parte aérea (PP), em t ha-1. A área útil de coleta foi de $4 \mathrm{~m}^{2}$ $(2 \times 2 \mathrm{~m})$, sendo o ponto de amostragem do solo posicionado no centro da mesma. As amostragens foram realizadas em 9/8/2002.

A análise descritiva inicial foi efetuada no SAS (Schlotzhaver \& Littell, 1997), e a geoestatística, no $\mathrm{GS}^{+}$(Robertson, 1998). Para testar o tipo de distribuição de freqüência dos atributos, foram utilizados os parâmetros referentes ao teste W, de Shapiro e Wilk, fornecidos pelo SAS. Foi utilizada a validação cruzada para avaliar a proposição do melhor modelo do semivariograma ajustado, seus parâmetros e o tamanho da vizinhança que proporcionou o melhor ajuste. Assim, o semivariograma selecionado foi o que apresentou o maior coeficiente de correlação, quando avaliada, para cada um deles, a regressão entre seus valores observados e estimados (Robertson, 1998; Silva et al., 2003). Dados de alguns atributos apresentaram tendência, aos quais foi aplicada a técnica do Refinamento pela Mediana, conforme preconizado por Silva et al. (2003), descrita a seguir: (a) os dados foram dispostos de acordo com sua posição original na malha amostral; (b) foi calculada a mediana correspondente a cada linha e coluna; e (c) foram subtraídos, de cada valor amostrado, os valores da mediana da linha e da coluna em que se encontrou esse valor, adicionando-lhe o valor da mediana de todo o conjunto de dados. Dessa forma, pôde-se calcular novamente o semivariograma com os resíduos originados desse procedimento. A análise da dependência espacial foi efetuada segundo a expressão (Robertson, 1998):

$$
\mathrm{ADE}=\left[\mathrm{C} /\left(\mathrm{C}+\mathrm{C}_{0}\right)\right] 100
$$

em que ADE é o estimador da dependência espacial (\%); C, a variância estrutural; e $\mathrm{C}+\mathrm{C}_{0}$, o patamar (Robertson, 1998; Landim, 1998). Assim, a interpretação proposta para ADE foi a seguinte: (a) $\mathrm{ADE} \leq 25 \%$ indica variável espacial fracamente dependente; (b) $25 \%<\mathrm{ADE} \leq 75 \%$, moderadamente dependente; e (c) $\mathrm{ADE}>75 \%$, fortemente dependente. $\mathrm{Na}$ confecção do mapa de krigagem foi utilizado o programa computacional GS+ (Robertson, 1998).

\section{RESULTADOS E DISCUSSÃO}

Os valores das frações granulométricas permitem classificar o perfil como textura argilosa (Embrapa, 1999). Entretanto, a distribuição granulométrica no perfil do solo em questão não pode ser confundida com a classe textural do solo da área, que, de acordo com Demattê (1980), se refere à textura argila pesada. A produtividade média de grãos $\left(2.594 \mathrm{t} \mathrm{ha}^{-1}\right)$ pôde ser justificada pelo fornecimento adequado de água às plantas, dado pelo sistema de irrigação utilizado.

De acordo com Pimentel-Gomez \& Garcia (2002), a variabilidade de um atributo pode ser classificada de acordo com a magnitude do seu coeficiente de variação, que pode ser: (a) baixa, quando menor que $10 \%$; (b) média, quando entre 10 e $20 \%$; (c) alta, quando entre 20 e $30 \%$; e (d) muito alta, se maior que $30 \%$. Assim, a variabilidade dos atributos do solo (Quadro 2) foi, para a fração argila, de 5,9 \% (ARG1) e 6,9 \% (ARG2), sendo ambas as camadas classificadas como baixas. Araújo (2002) e Barbieri et al. (2002) também encontraram variabilidade semelhante à desses dados, diferentemente de Gonçalves \& Folegatti (2002) e Roque et al. (2002), que encontraram variabilidade considerada média. Para a fração silte, ela foi de 15,8 \% (SIL1) e 16,9 \% (SIL2), classificada como média em ambos os casos. Situações parecidas também foram observadas por Barbieri et al. (2002) e Gonçalves \& Folegatti (2002), o que não ocorreu em trabalhos como os de Macedo et al. (1998) e Roque et al. (2002), nos quais a variabilidade foi alta. Para a fração areia, ela foi de 10,5 \% (ARE1) e 11,6 \% (ARE2), classificada como média nos dois casos. Variabilidade semelhante foi observada também nos trabalhos de Gonçalves \& Folegatti (2002) e Rabah (2002), porém trabalhos como os de Souza et al. (1997) e Roque et al. (2002) apresentaram baixa variabilidade para esse atributo. Não houve grande diferença no perfil ARG1 e ARG2 (1 \%), entre SIL1 e SIL2 (1,1 \%) e entre ARE1 e ARE2 (1,1\%). Esse fato se deve praticamente à variabilidade natural que os dados apresentaram, quando submetidos à repetição, conforme a metodologia aplicada.

A variabilidade dos atributos da planta (Quadro 2) foi alta, com valores de 21,2 \% (PP) e 23,8\% (PG). Em relação à $\mathrm{PG}$, o alto valor de $23,8 \%$ foi semelhante ao encontrado por Gonçalves et al. (1998) quando 
Quadro 2. Medidas estatísticas descritivas e distribuição de freqüência dos atributos da cultura do feijoeiro e de um Latossolo Vermelho distroférrico de Selvíria (MS) sob plantio direto

\begin{tabular}{|c|c|c|c|c|c|c|c|c|c|c|c|c|c|}
\hline \multirow{3}{*}{ Atributo $^{(1)}$} & \multicolumn{12}{|c|}{ Medida estatística descritiva } & \multirow{3}{*}{$\begin{array}{l}\text { Distribui } \\
\text { ção de } \\
\text { freqüên- } \\
\text { cia }^{(2)}\end{array}$} \\
\hline & \multirow[t]{2}{*}{ Média } & \multirow[t]{2}{*}{ Mediana } & \multirow[t]{2}{*}{ Moda } & \multirow{2}{*}{$\begin{array}{l}\text { Erro- } \\
\text { padrão da } \\
\text { média }\end{array}$} & \multicolumn{3}{|c|}{ Valor } & \multirow{2}{*}{$\begin{array}{l}\text { Desvio- } \\
\text { padrão }\end{array}$} & \multirow[t]{2}{*}{ Variância } & \multicolumn{3}{|c|}{ Coeficiente } & \\
\hline & & & & & Mín & Máx & $\begin{array}{l}\text { Ampli- } \\
\text { tude }\end{array}$ & & & $\begin{array}{c}\text { Variação } \\
(\%)\end{array}$ & Curtose & $\underset{\text { tria }}{\text { Assime- }}$ & \\
\hline \multicolumn{14}{|c|}{ Argila } \\
\hline ARG1 $\left(\mathrm{g} \mathrm{kg}^{-1}\right)$ & 469 & 466 & 474 & 2,366 & 420 & 540 & 120 & $2,749.10^{1}$ & $7,55610^{2}$ & 5,9 & $-0,568$ & 0,421 & $\mathrm{~L}$ \\
\hline $\operatorname{ARG} 2\left(\mathrm{~g} \mathrm{~kg}^{-1}\right)$ & 482 & 479 & 471 & 2,844 & 415 & 559 & 144 & $3,305.10^{1}$ & $1,09210^{3}$ & 6,9 & $-0,647$ & 0,112 & $\mathrm{~N}$ \\
\hline \multicolumn{14}{|c|}{ Silte } \\
\hline SIL1 ( $\left.\mathrm{g} \mathrm{kg}^{-1}\right)$ & 166 & 170 & 191 & 2,256 & 114 & 230 & 116 & $2,621.10^{1}$ & $6,87110^{3}$ & 15,8 & $-0,724$ & $-0,093$ & $?$ \\
\hline SIL2 $\left(\mathrm{g} \mathrm{kg}^{-1}\right)$ & 162 & 161 & 167 & 2,355 & 109 & 239 & 130 & $2,736.10^{1}$ & $7,48610^{3}$ & 16,9 & $-0,359$ & 0,248 & $\mathrm{~N}$ \\
\hline \multicolumn{14}{|c|}{ Areia } \\
\hline $\operatorname{ARE} 1\left(\mathrm{~g} \mathrm{~kg}^{-1}\right)$ & 363 & 373 & 406 & 3,286 & 286 & 416 & 130 & $3,818.10^{1}$ & $1,45810^{3}$ & 10,5 & $-1,217$ & $-0,283$ & $?$ \\
\hline $\operatorname{ARE} 2\left(\mathrm{~g} \mathrm{~kg}^{-1}\right)$ & 353 & 363 & 294 & 3,517 & 253 & 417 & 164 & $4,087.10^{1}$ & $1,67010^{3}$ & 11,6 & $-1,090$ & $-0,428$ & $?$ \\
\hline \multicolumn{14}{|c|}{ Atributos da planta } \\
\hline $\mathrm{PP}\left(\mathrm{t} \mathrm{ha} \mathrm{a}^{-1}\right)$ & 1,989 & 1,975 & 2,225 & $3,62210^{-2}$ & $8,45010^{-1}$ & 2,997 & 2,152 & $4,20810^{-1}$ & $1,77110^{-1}$ & 21,2 & $8,672.10^{-2}$ & $-6,145.10^{-2}$ & $\mathrm{~N}$ \\
\hline PG $\left(t h^{-1}\right)$ & 2,594 & 2,601 & 2,628 & $5,32010^{-2}$ & $9,55010^{-1}$ & 4,197 & 3,242 & $6,18210^{-1}$ & $3,8211^{-1}$ & 23,8 & $-5,967 \cdot 10^{-2}$ & $-2,807.10^{-2}$ & $\mathrm{~N}$ \\
\hline
\end{tabular}

(1) ARG1: argila da camada de 0,0-0,10 m; ARG2: argila da camada de 0,10-0,20 m; SIL1: silte da camada de 0,0-0,10 m; SIL2: silte da camada de 0,10-0,20 m; ARE1: areia da camada de 0,0-0,10 m; ARE2: areia da camada de 0,10-0,20 m; PP: produção da palha; PG: produção de grãos do feijoeiro. ${ }^{(b)} \mathrm{N}$ : distribuição normal, L: distribuição lognormal, ?: distribuição de freqüência desconhecida.

utilizaram o preparo do solo com aração (24\%). Entretanto, foi superior àquele desses autores quando usaram o preparo do solo com escarificação (16\%), assim como ao de Carvalho (2004), que foi de $15 \%$.

Empregando-se a classificação do coeficiente de correlação entre duas variáveis (Sharp, s/d), os pares de atributos apresentaram a seguinte classificação: (a) extra-alto: $\mathrm{PG} \times \mathrm{PP}(\mathrm{r}=0,85)$; (b) altos: ARG1 x ARG2 ( $r=0,68)$ e SIL1 x SIL2 ( $r=0,62)$; (c) moderados: ARE1 x ARG2 $(r=0,41)$, ARE1 x SIL1 $(r=-0,41)$ e ARE1 x ARE2 $(r=0,56)$; $(d)$ baixos: ARE1 $x$ SIL2 $(r=-0,30)$, ARE2 x SIL1 $(r=-0,28)$, ARE2 x SIL2 $(r=-0,24)$, PP x ARG1 $(r=-0,25)$, PG x ARG1 $(r=-0,27)$ e PG x ARE2 $(r=-0,21)$; e (e) nulos: ARG1 x SIL1, ARG2 x SIL1, ARG1 x SIL2, ARG2 x SIL2, ARG1 x ARE1, ARG1 x ARE2, ARG2 x ARE2, ARG2 x PP, SIL1 x PP, SIL2 x PP, ARE1 x PP, ARE2 x PP, ARG2 x PG, SIL1 x PG, SIL2 x PG e ARE1 x PG, os quais tiveram os valores extremos do coeficiente de correlação entre -0,02 (ARG1 x SIL1) e -0,17 (SIL2 x PG) (Quadro 3). Devese observar que Landim (1998) não deixou claramente explicitada a existência de afinidade entre a magnitude do coeficiente de correlação, entre duas variáveis, e a provável existência de semivariograma cruzado, que permitiria a realização da cokrigagem. Em relação ao presente estudo, pode-se inferir que provavelmente exista semivariograma cruzado entre os seguintes atributos: ARG1 x ARG2, SIL1 x SIL2 e PG x PP. Na oportunidade, vale ressaltar que Freddi (2003) observou excelente semivariograma cruzado, obtido entre a produtividade de grãos do feijoeiro e o $\mathrm{pH}$ do solo, com coeficiente de determinação espacial $\left(\mathrm{r}^{2}\right)$ de 0,806 , os quais, no entanto, apresentaram o baixo coeficiente de correlação (não-espacial) de 0,298. Portanto, sabendo da evidência de que baixos coeficientes de correlação, entre duas variáveis, não invalidam a hipótese de existência de semivariograma cruzado entre elas, pode-se perfeitamente também inferir, para o presente estudo, que provavelmente haja a possibilidade de cokrigagem entre todos os pares de atributos que tiveram coeficientes de correlação classificados como moderados, baixos e nulos.

A análise da variabilidade espacial dos atributos estudados (Quadro 4) evidenciou que a maioria deles apresentou dependência espacial (ARG1, ARG2, SIL1, SIL2, ARE1 e PG). Em contrapartida, ARE2 e PP resultaram em efeito pepita puro, não havendo, portanto, manifestação espacial neles. Assim, a constatação da referida dependência espacial evidenciou que a distribuição destes atributos no espaço não é aleatória, uma vez que todos apresentaram valores moderados de ADE, os quais variaram entre $50 \%$ (SIL2) e $75 \%$ (ARG2), diferentemente dos elevados valores observados na literatura (Souza et al., 1997; Barbieri et al., 2002; Gonçalves \& Folegatti, 2002; Carvalho, 2004).

O modelo esférico de semivariograma ajustou-se aos teores da fração argila (ARG1 e ARG2) (Quadro 4). Ajustes desse modelo foram também obtidos por Beraldo et al. (2000) e Gonçalves \& Folegatti (2002). Por sua vez, Rabah (2002) e Roque et al. (2002) obtiveram ajustes do modelo exponencial. Para a fração silte (SIL1 e SIL2), o modelo ajustado foi o exponencial, igualmente aos ajustes observados por 
Gonçalves et al. (2000) e Roque et al. (2002) em seus trabalhos. Em contrapartida, observou-se que nos trabalhos de Vieira (1997) e Salviano et al. (1998) os modelos ajustados foram diferentes, sendo eles esférico e gaussiano, respectivamente. Já para fração areia (ARE1 e ARE2) obteve-se ajuste apenas para (ARE1), utilizando o modelo esférico. Modelo de ajuste semelhante a este também foi obtido por Beraldo et al. (2000) e por Gonçalves \& Folegatti (2002), diferentemente dos observados por Barbieri et al. (2002) e Roque et al. (2002), que obtiveram ajuste exponencial. Para o atributo (ARE2), ocorreu o efeito pepita puro. Com relação aos atributos da planta (PG e PP), não houve ajuste para a PP que apresentou efeito pepita puro; já a PG ajustou-se melhor com o modelo gaussiano, o que também foi observado nos trabalhos de Gonçalves et al. (1998) e Carvalho (2004).

O alcance da dependência espacial da fração argila (Quadro 4) foi de 86,2 m (ARG1) e 87,4 m (ARG2). Esses valores foram superiores aos apresentados por Gonçalves \& Folegatti (2002) e Roque et al. (2002), e inferiores aos de Barbieri et al. (2002) e Rabah (2002). Os alcances da dependência espacial da fração silte foram de 19,8 m (SIL1) e 85,5 m (SIL2), valores estes superiores aos de Vieira (1997) e Roque et al. (2002); já em comparação aos valores obtidos por Gonçalves et al. (2001) e Barbieri et al. (2002), a amplitude entre

Quadro 3. Matriz de correlação entre a produtividade do feijoeiro e os atributos de um Latossolo Vermelho distroférrico de Selvíria (MS)

\begin{tabular}{|c|c|c|c|c|c|c|c|c|}
\hline \multirow{2}{*}{ Atributo $^{(1)}$} & \multicolumn{8}{|c|}{ Coeficiente de correlação } \\
\hline & ARG 1 & $\mathrm{ARG} 2$ & SIL1 & SIL2 & ARE1 & ARE2 & $\mathbf{P P}$ & PG \\
\hline ARG1 & - & & & & & & & \\
\hline ARG2 & 0,681 & - & & & & & & \\
\hline SIL1 & $-0,021$ & $-0,070$ & - & & & & & \\
\hline SIL2 & 0,141 & 0,065 & 0,622 & - & & & & \\
\hline ARE1 & $-0,124$ & 0,409 & $-0,407$ & $-0,302$ & - & & & \\
\hline ARE2 & 0,077 & 0,109 & $-0,283$ & $-0,243$ & 0,560 & - & & \\
\hline PP & $-0,246$ & $-0,166$ & $-0,022$ & $-0,094$ & $-0,095$ & $-0,157$ & - & \\
\hline$P G$ & $-0,271$ & $-0,134$ & $-0,120$ & $-0,172$ & $-0,110$ & $-0,207$ & 0,849 & - \\
\hline
\end{tabular}

(1) ARG1: argila da camada de 0,0-0,10 m; ARG2: argila da camada de 0,10-0,20 m; SIL1: silte da camada de 0,0-0,10 m; SIL2: silte da camada de 0,10-0,20 m; ARE1: areia da camada de 0,0-0,10 m; ARE2: areia da camada de 0,10-0,20 m; PP: produção da palha; PG: produção de grãos do feijoeiro.

Quadro 4. Parâmetros dos semivariogramas ajustados aos atributos da cultura do feijoeiro e de um Latossolo Vermelho distroférrico de Selvíria (MS)

\begin{tabular}{|c|c|c|c|c|c|c|c|c|c|}
\hline Atributo $^{(1)}$ & Modelo $^{(2)}$ & $\begin{array}{c}\text { Efeito } \\
\text { pepita } \\
\text { (Co) }\end{array}$ & $\begin{array}{c}\text { Patamar } \\
(\mathrm{Co}+\mathrm{C})\end{array}$ & $\begin{array}{c}\text { Variância } \\
\text { estrutural } \\
\text { (C) }\end{array}$ & Alcance & $\mathbf{r}^{2}$ & $\mathrm{SQR}^{(3)}$ & $\mathrm{ADE}^{(4)}$ & $\begin{array}{c}\text { Classe de } \\
\text { dependência } \\
\text { espacial }\end{array}$ \\
\hline & & & & & $\mathrm{m}$ & & & $\%$ & \\
\hline ARG1 $\left(\mathrm{g} \mathrm{kg}^{-1}\right)$ & esf & $1,880.10^{2}$ & $1,880.10^{2}$ & $1,880.10^{2}$ & 86,2 & 0,910 & $1,993.10^{4}$ & 71,5 & moderada \\
\hline ARG2 $\left(\mathrm{g} \mathrm{kg}^{-1}\right)$ & esf & $2,730.10^{2}$ & $1,090.10^{3}$ & $8,170.10^{2}$ & 87,4 & 0,932 & $4,492.10^{4}$ & 75,0 & forte \\
\hline SIL1 $\left(\mathrm{g} \mathrm{kg}^{-1}\right)$ & $\exp$ & $7,990.10^{1}$ & $3,175.10^{2}$ & $2,376.10^{2}$ & 19,8 & 0,332 & $1,055.10^{4}$ & 74,8 & moderada \\
\hline SIL2 $\left(\mathrm{g} \mathrm{kg}^{-1}\right)$ & $\exp$ & $2,380.10^{2}$ & $4,761.10^{2}$ & $2,381.10^{2}$ & 85,5 & 0,626 & $1,152.10^{4}$ & 50,0 & moderada \\
\hline $\operatorname{ARE} 1\left(\mathrm{~g} \mathrm{~kg}^{-1}\right)$ & esf & $4,650.10^{2}$ & $1,181.10^{3}$ & $7,160 \cdot 10^{2}$ & 103,1 & 0,898 & $4,715.10^{4}$ & 60,6 & moderada \\
\hline $\operatorname{ARE} 2\left(\mathrm{~g} \mathrm{~kg}^{-1}\right)$ & epp & $2,715.10^{3}$ & - & - & - & - & - & - & - \\
\hline PG $\left(t h a^{-1}\right)$ & gau & $8,410 \cdot 10^{-2}$ & $2,452.10^{-1}$ & $1,611.10^{-1}$ & 29,8 & 0,888 & $3,491 \cdot 10^{-3}$ & 65,7 & moderada \\
\hline $\mathrm{PP}\left(\mathrm{t} \mathrm{ha}^{-1}\right)$ & epp & $1,798 \cdot 10^{-1}$ & - & - & - & - & - & - & - \\
\hline
\end{tabular}

(1) ARG1: argila da camada de 0,0-0,10 m; ARG2: argila da camada de 0,10-0,20 m; SIL1: silte da camada de 0,0-0,10 m; SIL2: silte da camada de 0,10-0,20 m; ARE1: areia da camada de 0,0-0,10 m; ARE2: areia da camada de 0,10-0,20 m; PP: produção da palha; PG: produção de grãos do feijoeiro. ${ }^{(2)}$ esf: esférico, exp: exponencial, gau: gaussiano e epp: efeito pepita puro. ${ }^{(3)}$ SQR: soma dos quadrados dos resíduos. ${ }^{(4)} \mathrm{ADE}$ : avaliador da dependência espacial. 
os alcances do (SIL1) e (SIL2) foi muito semelhante, porém superior à de Gonçalves et al. (2000). O alcance da dependência espacial da (ARE1) foi de 103,1 m, superior aos de Gonçalves \& Folegatti (2002) e Roque et al. (2002) e inferior aos de Barbieri et al. (2002) e Rabah (2002). O alcance da dependência espacial da produtividade de grãos (PG) foi de $29,8 \mathrm{~m}$, superior ao de Gonçalves et al. (1998) e Carvalho (2004), que foi de 10 e $20 \mathrm{~m}$, respectivamente.

As validações cruzadas, representantes dos semivariogramas ajustados, evidenciaram que os atributos do solo (ARG1, ARG2, SIL1, SIL2 e ARE1) tiveram bom desempenho, relativamente superior àquele da planta (PG). Isso porque foi observada maior tendência, em relação aos atributos do solo, de o coeficiente linear (a) tender a zero e de o angular (b) tender a um, assim como os mais elevados coeficientes de correlação. Já em relação ao PG, o coeficiente b foi o que mais se distanciou de 1 , assim como revelou o menor coeficiente de correlação entre todos os atributos $(r=0,36)$. Pode-se também observar que todos os atributos apresentaram maiores valores do erro-padrão estimado do que do observado, semelhante ao constatado por Marx et al. (1988).

No que se refere aos atributos dos quais foi extraída a tendência dos dados (Figura 1c, d, e), uma vez que não foram efetuadas as respectivas retrotransformações, pode-se notar, no mapa de krigagem, que seus valores podem ser negativos. Os valores mínimos e máximos dos atributos contidos no quadro 2 podem ser atribuídos àqueles que apresentaram tendência. Assim, em relação ao SIL1, seus valores extremos (-24 e $23 \mathrm{~g} \mathrm{~kg}^{-1}$ ) provavelmente sejam de 114 e $230 \mathrm{~g} \mathrm{~kg}^{-1}$. De forma semelhante, em relação ao SIL2, seriam de - 16 e $20 \mathrm{~g} \mathrm{~kg}^{-1}$ (irreais) e 109 e $239 \mathrm{~g} \mathrm{~kg}^{-1}$ (inferidos); assim como, em relação ao ARE1, de -9 e $38 \mathrm{~g} \mathrm{~kg}^{-1}$ (irreais) e 286 e $416 \mathrm{~g} \mathrm{~kg}^{-1}$ (inferidos).

Analisando o Quadro 3 e a Figura 1, tendo-se por base tanto o observado por Landim (1998) como o obtido por Freddi (2003), constatou-se, em relação aos atributos que apresentaram alta correlação (ARG1 x ARG2 e SIL1 x SIL2), evidente correlação espacial positiva entre eles. Assim, os mapas de krigagem, efetuados entre ARG1 e ARG2, se apresentaram de forma muito semelhante, com valores mínimos e máximos coincidindo praticamente nas mesmas regiões, visto que a correlação entre elas foi positiva. Basicamente, observou-se o mesmo em relação a SIL1 e SIL2. Por outro lado, em relação aos atributos que apresentaram moderada correlação (ARE1 x ARG2 e ARE1 x SIL1), foi observada a mesma tendência, direta ou indiretamente, conforme a natureza positiva ou negativa entre eles. Ainda que com certo grau de reserva, o mesmo fato foi observado para aqueles que apresentaram baixa correlação (ARE1 x SIL2 e PG x ARG1). Dessa forma, as correlações espaciais parecem existir independentemente dos valores das correlações lineares, sejam eles altos ou baixos entre os atributos. (a)

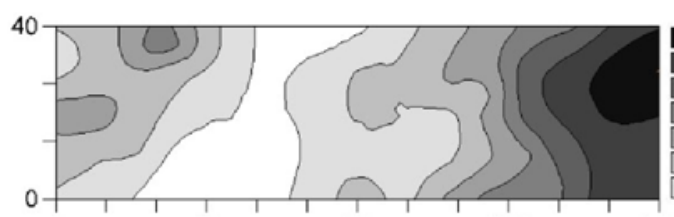

(c)

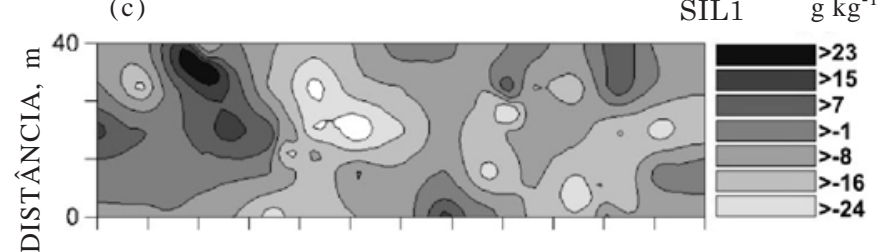

(b)

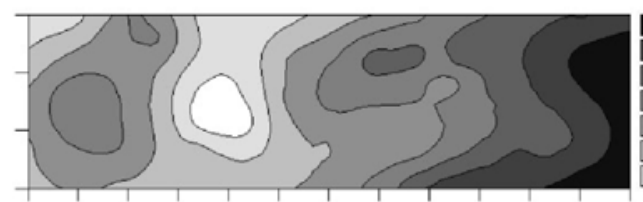

(d)

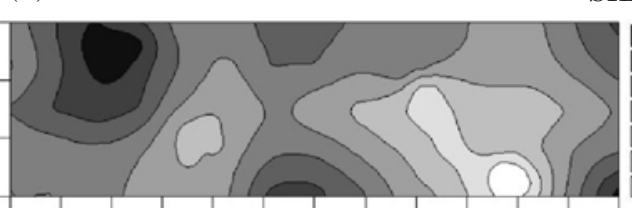

SIL2 $\quad \mathrm{g} \mathrm{kg}^{-1}$

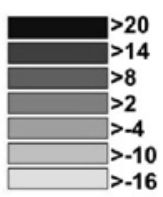

(f)

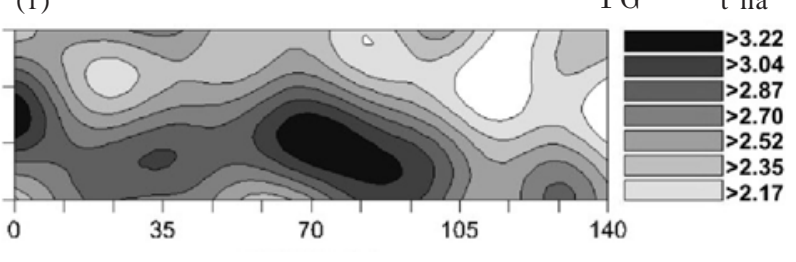

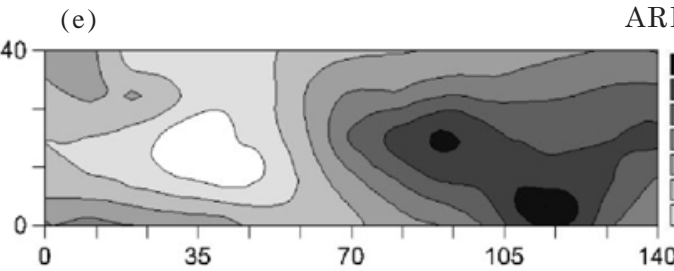

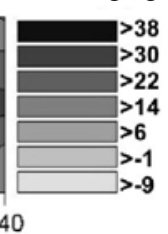

DISTÂNCIA, m

(a) ARG1: argila da camada 0,0-0,10 m; (b) ARG2: argila da camada 0,10-0,20 m; (c) SIL1: silte da camada 0,0-0,10 m; (d) SIL2: silte da camada 0,10-0,20 m; (e) ARE1: areia da camada 0,0-0,10 m, e (f) PG: produção de grãos do feijoeiro.

Figura 1. Mapas de krigagem das frações granulométricas e da produtividade do feijoeiro num Latossolo Vermelho distroférrico de Selvíria (MS). 


\section{CONCLUSÕES}

1. Apenas $7,3 \%$ da produtividade de grãos de feijão foi explicada pelo teor de argila da camada superficial do solo.

2. As frações granulométricas do solo e a produtividade de grãos do feijoeiro não variaram aleatoriamente, seguindo padrões espaciais bem definidos, relacionados ora direta, ora inversamente.

3. Neste estudo, as evidências mostraram indicativos de que baixos valores de coeficiente de correlação, entre dois atributos quaisquer, não invalidam a hipótese de ocorrência de apreciável correlação espacial entre eles.

\section{AGRADECIMENTO}

Ao Conselho Nacional de Desenvolvimento Científico e Tecnológico (CNPq), pelo auxílio financeiro.

\section{LITERATURA CITADA}

ARAUJO, A.V. Variabilidade espacial de propriedades químicas e granulométricas do solo na definição de zonas homogêneas de manejo. Jaboticabal, Universidade Estadual Paulista, 2002. 80p. (Tese de Mestrado)

BARBIERI, D.M.; SOUZA, Z.M.; VIEIRA, J.C.; MARQUES JUNIOR, J.; CORÁ, J.E. \& PEREIRA, G.T. Dependência espacial de atributos granulométricos em um Latossolo Vermelho Eutroférrico sob cultivo de cana-de-açúcar na Região de Jaboticabal (SP). In: REUNIÃO BRASILEIRA DE MANEJO E CONSERVAÇÃO DO SOLO E DA ÁGUA, 14., Cuiabá, 2002. Anais. Cuiabá, Sociedade Brasileira de Ciência do Solo, 2002. CD-ROM

BERALDO, L.M.; G.; CORÁ, J.E.; MARQUES JUNIOR, J.; PEREIRA, G.T. \& POCAY, V.G. Definição de zonas homogêneas de manejo através da caracterização da variabilidade espacial da textura do solo. In: REUNIÃO BRASILEIRA DE MANEJO E CONSERVAÇÃO DO SOLO E DA ÁGUA, 13., Ilhéus, 2000. Anais. Ilhéus, Sociedade Brasileira de Ciência do Solo, 2000. CD-ROM

BHATTI, A.U.; MULLA, D.J. \& FRAZIER, B.E. Estimulation of soil properties and wheat yields on complex eroded hills using geoestatistics and thematic mapper images. Remote Sens., 37:181-191, 1991.

CARVALHO, G.J. Correlação linear e espacial da produtividade do feijão (Phaseolus vulgaris L.) com a resistência mecânica à penetração de um Latossolo Vermelho distroférrico sob plantio direto. Ilha Solteira, Universidade Estadual Paulista, 2004. 50p. (Monografia de Graduação)

DEMATTÊ, J.L.I. Levantamento detalhado de solos do "Campus Experimental de Ilha Solteira". Piracicaba, Escola Superior de Agricultura "Luiz de Queiroz", 1980. 44p.
EMPRESA BRASILEIRA DE PESQUISA AGROPECUÁRIA EMBRAPA. Centro Nacional de Pesquisa de Solos. Sistema brasileiro de classificação de solos. Rio de Janeiro, 1999. 412p.

EMPRESA BRASILEIRA DE PESQUISA AGROPECUÁRIA EMBRAPA. Centro Nacional de Pesquisa de Solos. Manual de métodos de análise do solo. 2.ed. Rio de Janeiro, 1997.

FAHL, J.I.; CAMARGO, M.B.P.; PIZZINATTO, M.A.; BETTI, J. A.; MELO, A.M.T.; DeMARIA, I.C. \& FURLANI, A.M.C. Instruções agrícolas para as principais culturas econômicas. 6.ed. Campinas, Instituto Agronômico de Campinas, 1998. p.281-282. (Boletim Técnico 200)

FREDDI, O.S. Variabilidade especial da produtividade do feijão (Phaseolus vulgaris L.) e de atributos químicos de um Latossolo Vermelho distroférrico de Selvíria (MS) sob preparo convencional e plantio direto. Ilha Solteira, Universidade Estadual Paulista, 2003. 154p. (Tese de Mestrado)

GEE, G.W. \& BAUDER, J.W. Particle size analysis. In: KLUTE, A., ed. Methods of soil analysis. Physical and mineralogical methods. 2.ed. Madison, American Society of Agronomy, 1986. p.383-411.

GONÇALVES, A.C.A. Variabilidade espacial de propriedades físicas do solo para fins de manejo da irrigação. Botucatu, Escola Superior de Agricultura Luiz de Queiroz, 1997. 118p. (Tese de Doutorado)

GONÇALVES, A.C.A. \& FOLEGATTI, M.V. Correlação espacial entre retenção de água e textura do solo para fins de manejo de irrigação. Eng. Agric., 22:296-303, 2002.

GONÇALVES, A.C.A.; FOLEGATTI, M.V. \& MATA, J.D.V. Análises exploratórias e geoestatística da variabilidade de propriedades físicas de um Argissolo Vermelho. Acta Sci., 23:1149-1157, 2001.

GONÇALVES, A.C.A.; MATA, J.D.V.; VIEIRA, S.R. \& FOLEGATTI, M.V. Variabilidade espacial de produtividade e de resistência à penetração em área irrigada, sob dois sistemas de preparo. In: AVANCES EN EL MANEJO DEL SUELO Y AGUA EN LA INGENIERÍA RURAL LATINOAMERICANA, La Plata, 1998. Anais. La Plata, Universidad Nacional de La Plata, 1998. p.113-119.

GONÇLVES, C.; ABREU, S.L.; SILVEIRA, M.J.; REICHERT, J.M. \& REINERT, D.J. Variabilidade espacial de propriedades hídricas e mecânicas de um Argissolo sob sistema de plantio direto. In: REUNIÃO BRASILEIRA DE MANEJO E CONSERVAÇÃO DO SOLO E DA ÁGUA,13., Ilhéus, 2000. Anais. Ilhéus, Sociedade Brasileira de Ciência do Solo, 2000. CD-ROM

GUERRA, A.J.T. Geomorfologia: uma atualização de bases e conceitos. 3.ed. Rio de Janeiro, Bertrand Brasil, 1998. $472 \mathrm{p}$.

LANDIM, P.M.B. Análise estatística de dados geológicos. São Paulo, Fundação Editora da UNESP (Ciência e Tecnologia), 1998. 226p.

MACEDO, J.R.; OTONNI FILHO, T.B. \& MENEGUELLI, N.A. Variabilidade de características físicas, químicas e físicohídricas em solo Podzólico Vermelho-Amarelo de Seropédica, RJ. Pesq. Agropec. Bras., 33:2043-2053, 1998. 
MARX, D.B.; GILMOUR, J.T.; SCOTT, H.D. \& FERGUSO, J.A. Effects of long-term water management in a humid regime on spatial variability of soil chemical status. Soil Sci., 145:188-193, 1988.

PIMENTEL-GOMEZ, F. \& GARCIA, C.H. Estatística aplicada a experimentos agronômicos e florestais: exposição com exemplos e orientações para uso de aplicativos. Piracicaba, FEALQ, 2002. 309p.

RABAH, F.A. Dependência espacial de atributos do solo obtidos por meio de semivariograma e autocorrelogramas. Botucatu, Universidade Estadual Paulista, 2002. 90p. (Tese de Doutorado)

ROBERTSON, G.P. GS GS $^{+}$Geostatistics for the environmental sciences. Michigan, Gamma Desing Software, 1998. 151p.

ROQUE, C.G.; CENTURION, J.F.; PEREIRA, G.T. \& BEUTLER, N. Variabilidade espacial de atributos físicos em Argissolo Vermelho Amarelo cultivado com seringueira. In: REUNIÃO BRASILEIRA DE MANEJO E CONSERVAÇÃO DO SOLO E DA ÁGUA, 14., Cuiabá, 2002. Anais. Cuiabá, Sociedade Brasileira de Ciência do Solo, 2002. CD-ROM

SALVIANO, A.A.C.; VIEIRA, S.R. \& SPAROVEK, G. Variabilidade espacial de atributos de solo e de Crotalaria juncea (L.) em área severamente erodida. R. Bras. Ci. Solo, 22:115-122, 1998.

SCHLOTZHAVER, S.D. \& LITTELL, R.C. SAS system for elementary statistical analysis. 2.ed. Cary, SAS, 1997. 441p.
SHARP. Instructor manual. Scientific computer, Model EL5500 II. Local, Sharp Eletronics Corporation. Japan, 199?. $196 \mathrm{p}$.

SILVA, V.R.; REICHERDT, J.M.; STORCK, L. \& FEIJÓ, S. Variabilidade espacial das características químicas do solo e produtividade de milho em um Latossolo VermelhoAmarelo distrófico arênico. R. Bras. Ci. Solo, 27:10131020, 2003.

SOUZA, L.S. Variabilidade espacial do solo em sistemas de manejo. Porto Alegre, Universidade Federal do Rio Grande do Sul, 1992. 162p. (Tese de Doutorado)

SOUZA, L.S.; COGO, N.P. \& VIEIRA, S.R. Variabilidade de propriedade físicas e químicas do solo em um pomar cítrico. R. Bras. Ci. Solo, 21:367-372, 1997.

VIEIRA, L.S.; SANTOS, P.C.T.C. \& VIEIRA, M.N.S. Solos: propriedade, classificação e manejo. Brasília, MEC/ ABEAS, 1988.154p. (Programa Agricultura nos Trópicos, v.2)

VIEIRA, S.R. Variabilidade espacial de argila, silte e atributos químicos em uma parcela experimental de um Latossolo Roxo de Campinas. Bragantia, 56:181-190, 1997.

ZIMBACK, C.R.L. \& CATANEO, A. Variabilidade espacial das características físicas de solos. In: AVANCES EM EL MANEJO DEL SUELOS Y AGUA EM LA INGENIERÍA RURAL LATINOAMERICANA, 1998, La Plata, Anais. La Plata, Universidad Nacional de La Plata, 1998. p.132-137. 